\title{
Division site selection linked to inherited cell surface wave troughs in mycobacteria
}

\author{
Haig A. Eskandarian ${ }^{1,2}$, Pascal D. Odermatt ${ }^{2}$, Joëlle X. Y. Ven ${ }^{1,2}$, Mélanie T. M. Hannebelle ${ }^{1,2}$, \\ Adrian P. Nievergelt ${ }^{2}$, Neeraj Dhar ${ }^{1}$, John D. McKinney ${ }^{1 \star \dagger}$ and Georg E. Fantner ${ }^{2 \star \dagger}$
}

\begin{abstract}
Cell division is tightly controlled in space and time to maintain cell size and ploidy within narrow bounds. In bacteria, the canonical Minicell (Min) and nucleoid occlusion (Noc) systems together ensure that division is restricted to midcell after completion of chromosome segregation'. It is unknown how division site selection is controlled in bacteria that lack homologues of the Min and Noc proteins, including mycobacteria responsible for tuberculosis and other chronic infections ${ }^{2}$. Here, we use correlated optical and atomic force microscopy ${ }^{3,4}$ to demonstrate that morphological landmarks (waveform troughs) on the undulating surface of mycobacterial cells correspond to future sites of cell division. Newborn cells inherit wave troughs from the (grand)mother cell and ultimately divide at the centre-most wave trough, making these morphological features the earliest known landmark of future division sites. In cells lacking the chromosome partitioning (Par) system, missegregation of chromosomes is accompanied by asymmetric cell division at off-centre wave troughs, resulting in the formation of anucleate cells. These results demonstrate that inherited morphological landmarks and chromosome positioning together restrict mycobacterial division to the midcell position.
\end{abstract}

Atomic force microscopy (AFM) has been used previously for static $^{5,6}$ or short-term time-lapse ${ }^{7}$ imaging of mycobacteria, primarily to study the impact of antibiotics and antimicrobial peptides on nanoscale features of the mycobacterial cell surface. Here, we use long-term time-lapse AFM to track cell growth and division over multiple generations in Mycobacterium smegmatis, a non-pathogenic relative of Mycobacterium tuberculosis (Fig. 1a, Supplementary Videos 1 and 2 and Supplementary Figs 1 and 2). Unexpectedly, we found that the cell surface undulates along the long axis (Fig. $1 \mathrm{~b}, \mathrm{c}$ ) in a roughly repeating waveform pattern with an average wavelength of $\sim 1.8 \mu \mathrm{m}$ (Supplementary Fig. 3). These morphological features are too small in amplitude $(\sim 100 \mathrm{~nm}$ from wave crest to wave trough) to resolve by conventional optical microscopy and they are morphologically distinct from the previously described 'division scars' ${ }^{8}$ (Supplementary Fig. 4, last panel, black arrow). Cell elongation is accompanied by an increase in wave trough number, as cells, on average, are born with three wave troughs and divide with four wave troughs after elongating by $2 \mu \mathrm{m}$ (Supplementary Table 1). In contrast, the distance between wave troughs does not scale with increasing cell length. Cells filamented with ciprofloxacin exhibit a greater number of wave troughs as a function of increased cell length (Supplementary Fig. 6). Conversely, blocking cell elongation with isoniazid (Supplementary Fig. 2a) prevents the formation of new wave troughs (Supplementary Fig. 7). While the undulating surface morphology is maintained in isoniazid-treated cells, height 48 increases along the cell length (Supplementary Figs 2b and 8), 49 possibly due to sustained metabolic activity.

In time-lapse series, we found that centrally located wave troughs correspond to future sites of cell division (Fig. 1c and Supplementary Figs 9-11). The centre-most wave trough is localized, on average, at $56 \%$ of the cell length relative to the old cell pole, ranging from $49 \%$ to $62 \%$ ( 25 th to 75 th percentiles, respectively). Remarkably, wave troughs that mark future division sites are already present at birth - they form near the cell poles in the mother, grandmother or great-grandmother cell and are passed on to the daughter cells at division (Fig. 2a,b, Supplementary Fig. 11 and Supplementary Table 1). On average, division at a wave trough occurs 1.3 generations after the trough is first established (Fig. $2 \mathrm{~b}$ and Supplementary Table 1), which corresponds to $\sim 4 \mathrm{~h}$ for cells growing with an average interdivision time of $\sim 3 \mathrm{~h}$ (Fig. 2c). Cell elongation gradually shifts the position of wave troughs towards the cell centre (Fig. 2a, Supplementary Video 2 and Supplementary Figs 4 and 11) . Inherited wave troughs localize to positions near the midcell $\sim 150$ min before cell cleavage. In cells inheriting multiple wave troughs, the amount of growth from each cell pole determines which wave trough is located closest to the midcell and becomes the division site. Depletion of RipA, a hydrolase essential for cleavage $^{10}$, results in chains of non-separated daughter cells; cells located internal to the chain (with no free ends) do not elongate and do not form new wave troughs, although they may still form septa within pre-existing wave troughs (Supplementary Video 3 and Supplementary Fig. 5).

In a microscope that combines optical (fluorescence) and low-noise AFM-based imaging ${ }^{4}$, time-lapse imaging of single cells revealed a sequence of morphological and molecular events leading up to cell division. Formation of the FtsZ contractile ring at midcell is thought to be the earliest event specifying the cell division site in rod-shaped bacteria ${ }^{1,11}$. In cells expressing FtsZ tagged with green fluorescent protein (GFP), we found that the FtsZ ring forms within a pre-existing wave trough near midcell (Fig. 3a). In cells growing with an average interdivision time of $190 \mathrm{~min}$, formation of the pre-divisional wave trough precedes formation of the FtsZ ring by $120 \mathrm{~min}$ on average. Cells filamented with mitomycin $\mathrm{C}$ exhibit the formation of multiple FtsZ rings at multiple wave troughs (Supplementary Fig. 12).

Formation of the FtsZ-GFP ring near midcell (Fig. 3a and Supplementary Video 4 ) is followed $\sim 30$ min later by the appearance of a co-localized 'pre-cleavage furrow' $(\sim 50 \mathrm{~nm}$ wide and $\sim 10 \mathrm{~nm}$ deep) in the AFM image, a distinctive topological feature that is too small to detect by optical microscopy (Fig. 3a, black arrows). This feature might correspond to the previously reported 'cell wall 
a
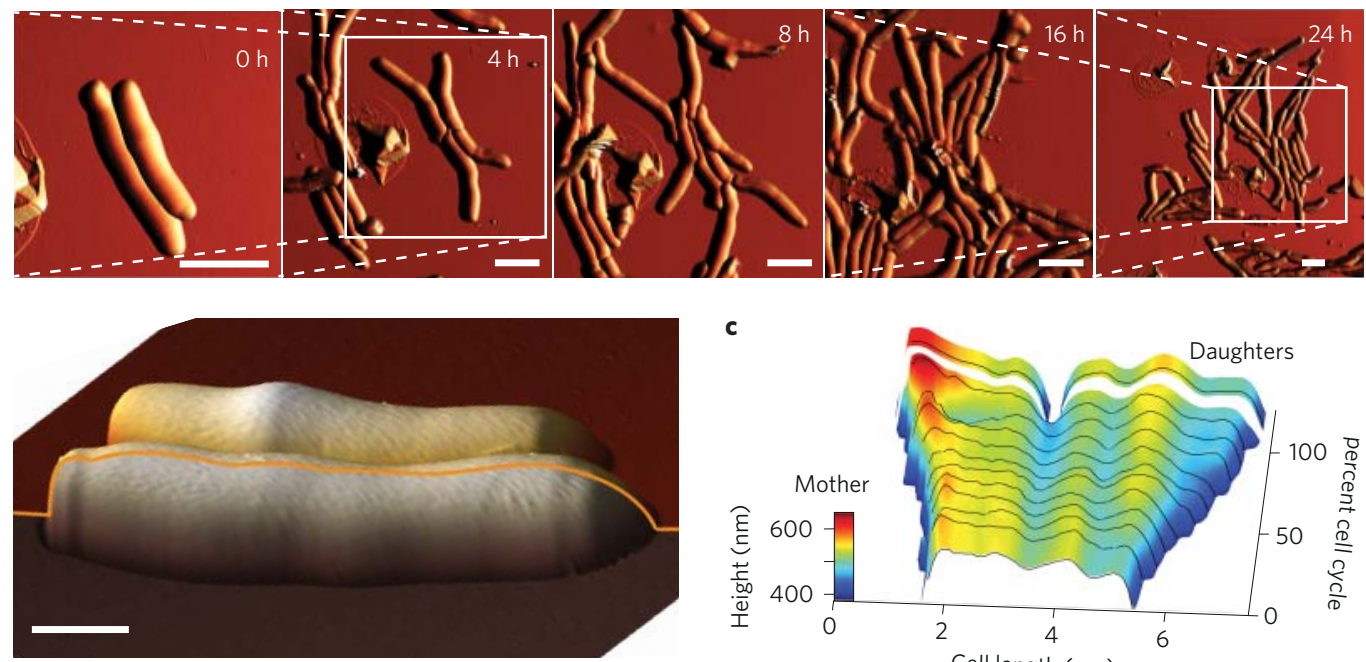

Figure 1 | Mycobacterial cells divide at cell surface wave troughs. a, Time series of three-dimensional AFM height images overlaid with AFM peak force error images for wild-type M. smegmatis. Scale bar, $3 \mu \mathrm{m}$. b. Mycobacterial surface topology. Yellow trace of the cell profile, highlighting the undulating mycobacterial surface morphology. Scale bar, $1 \mu \mathrm{m}$. c, Kymograph of the cell surface height of a representative cell from birth to division, showing that division occurs within the centre-most wave trough. In $\mathbf{b}$ and $\mathbf{c}$, images are representative of $n=270$.
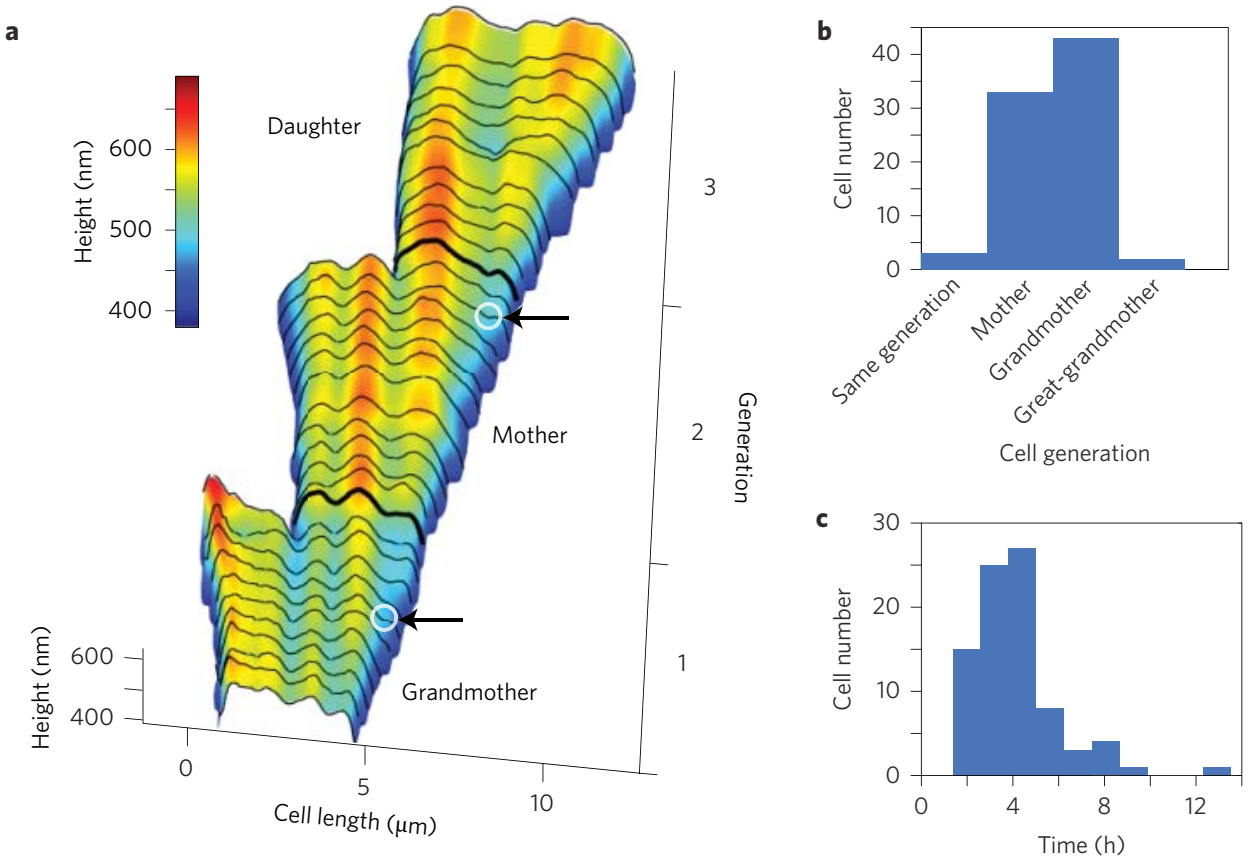

Figure 2 | Wave troughs are inherited from the (grand)mother cell. a, Kymograph of cell heights of one cell lineage over three consecutive generations (bottom to top). A wave trough formed in the grandmother cell (arrow) becomes the division site in the mother cell. Similarly, a wave trough formed in the

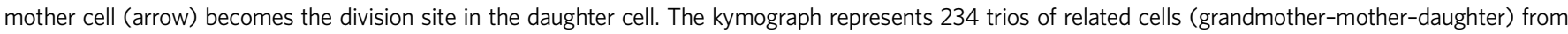
18 unrelated cell lineages. b. Distribution of generations from wave trough formation to cell cleavage. The wave trough where division occurs in the daughter

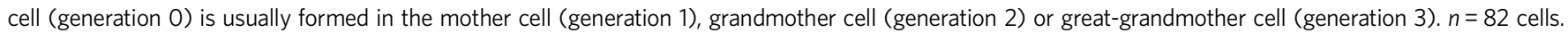
c, Distribution of time intervals from wave trough formation to cell cleavage. $n=82$ cells.

1 contractile ring' in Mycobacterium sp. JLS, although the latter has been described as a cell surface protrusion rather than an indentation ${ }^{7}$. The pre-cleavage furrow appears at around the same time as the early stages of septum formation, which we visualized by staining the cell membrane with the fluorescent dye FM4-64 (Fig. 3b, Supplementary Fig. 13 and Supplementary Videos 5 and 6). These events precede cytokinesis by $\sim 20 \mathrm{~min}$ in cells expressing the cytokinetic marker Wag31-GFP (Fig. 3c and Supplementary
Video 7). Cytokinesis is followed by a lag period of $\sim 40 \mathrm{~min} 9$ before physical cleavage of the sibling cells, signalled by an abrupt 10 deepening of the pre-cleavage furrow to $\sim 100 \mathrm{~nm}$ (Fig. 3 and 11 Supplementary Video 7).

Most mycobacterial cells inherit multiple wave troughs at birth, 13 yet only the centre-most wave trough is ultimately selected as the 14 division site. We asked whether off-centre wave troughs could 15 function as alternative sites of cell division in cells lacking the 16 

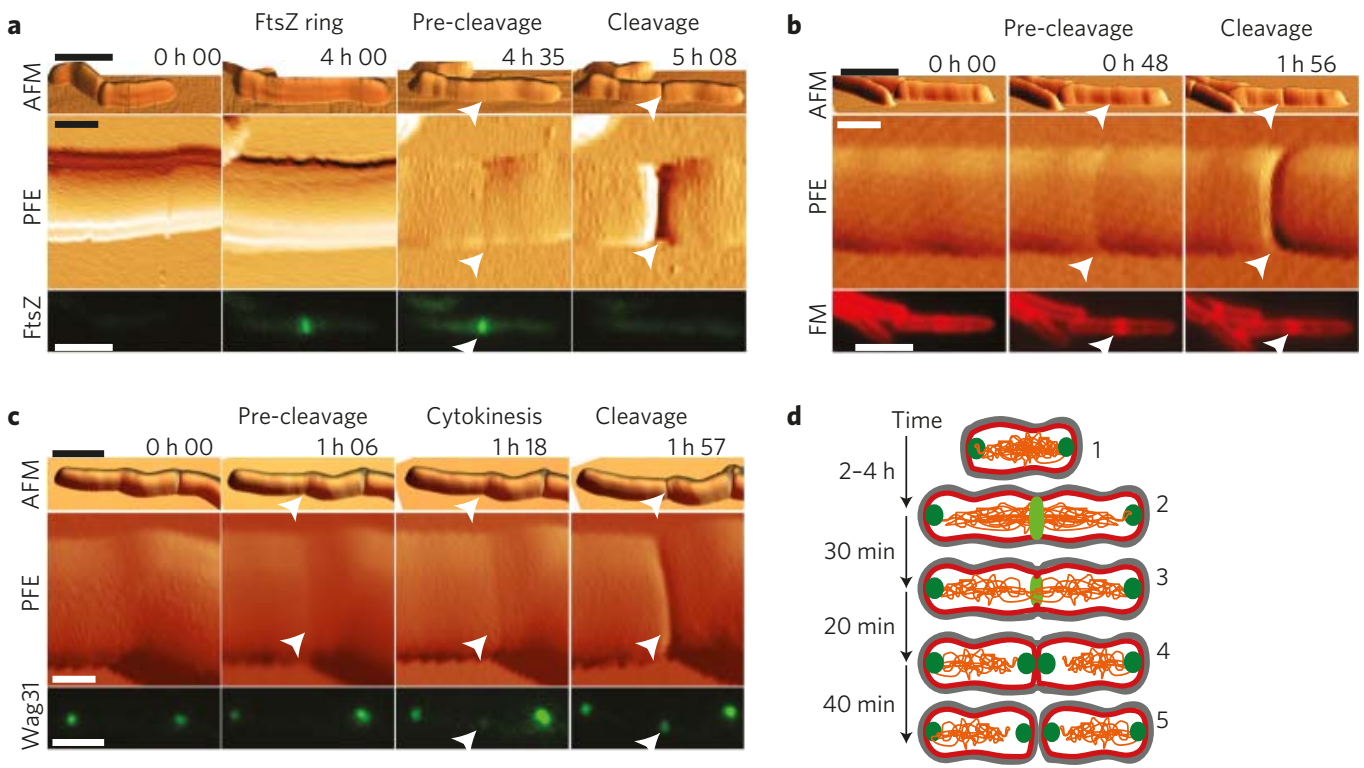

Figure 3 | Sequence of events from cell birth to cell division. Cells were imaged by correlated AFM (upper and middle panels) and fluorescence microscopy (bottom panels). Upper panels: three-dimensional representations of AFM topography images. Middle panels: AFM peak force error, which highlights the appearance of the pre-cleavage furrow and cleavage (white arrowheads). Numbers indicate time elapsed since birth. Scale bars (upper and bottom), $3 \mu \mathrm{m}$; (middle), $500 \mathrm{~nm}$. Images are representative of $n>10$. a, Cells expressing FtsZ-GFP. The FtsZ ring co-localizes with the pre-cleavage furrow (images are representative of $n=17$ ). $\mathbf{b}$, Cells with FM4-64-stained plasma membrane. Septum invagination co-localizes with the pre-cleavage furrow (images are representative of $n=15$ ). c, Cells expressing the cytokinesis marker Wag31-GFP. Cytokinesis is completed before cell cleavage (images are representative of $n=50$ ). $\mathbf{d}$, Schematic of the sequence of events culminating in cell division. At birth, Wag31-GFP (dark green) localizes exclusively at the cell poles (1). FtsZ-GFP (light green) forms a circumferential ring within the central wave trough (2-3). A pre-cleavage furrow then appears (indentation in the outer grey layer of the cell surface) and co-localizes with the FtsZ ring (3). Septum formation then proceeds and culminates in cytokinesis, which is marked by the appearance of a Wag31-GFP ring that co-localizes with the pre-cleavage furrow (4). Cell cleavage results in physical separation of the newborn sibling cells (5).

ParB chromosome-partitioning protein. Consistent with recent studies $^{12-14}$, we found that $\triangle$ par $B$ cells frequently undergo asymmetric cell divisions (Fig. 4a) within off-centre wave troughs (Fig. 4b,c and Supplementary Fig. 14). Divisions occurring at off-centre wave troughs are skewed towards the old or new cell pole ( $15 \%$ or $25 \%$, respectively), with the remainder of divisions $(60 \%)$ occurring at the centre-most wave trough. Divisions in $\triangle$ parB cells often occur in newly formed wave troughs (Supplementary Fig. 15).

Time-lapse fluorescence microscopy revealed that midcell divisions in $\triangle$ parB cells are associated with normal chromosome partitioning (Fig. 4d). We never observed divisions occurring at a local DNA maximum (Supplementary Figs 16 and 17). These results suggest that chromosomes might play a negative regulatory role in determining which wave trough is selected as the division site. Cells treated with the DNA gyrase inhibitor ciprofloxacin form elongated filaments with multiple wave troughs (Fig. 4e, first time point). Appearance of a pre-cleavage furrow in a filamented cell corresponds spatially to a local DNA minimum (Fig. 4e, arrows; Supplementary Fig. 6b and 6c).

Previous studies using $\mathrm{AFM}^{6,7,15}$, electron cryotomography ${ }^{16,17}$, or scanning electron microscopy ${ }^{8}$ identified a variety of bacterial surface features associated with initiation or completion of cell division. To the best of our knowledge, inherited morphological features associated with division site selection have not been identified until now. Although the well-characterized Min and Noc systems serve as negative regulators of FtsZ ring formation in evolutionarily divergent bacteria, emerging evidence suggests that these systems might not be responsible for initial specification of the division site per $s e^{1}$. Rather, these mechanisms may function at later steps to help ensure that the FtsZ ring forms only at an appropriate place (distant from the cell poles and membrane-tethered
DNA) and at the correct time relative to nucleoid segregation. What, 33 then, are the mechanisms responsible for specifying the future 34 division site? In Streptococcus pneumoniae, the MapZ protein 35 localizes as a circumferential band at midcell and sets the orientation 36 of the FtsZ ring ${ }^{18}$. However, most bacteria, including mycobacteria, 37 do not encode a MapZ homologue.

We show here that mycobacterial cell division occurs within 39 wave troughs on the undulating cell surface. Various bacterial 40 proteins are known to localize to negative ${ }^{19}$ or positive ${ }^{20}$ membrane 41 curvatures. Mycobacterial proteins that target curved membranes 42 within wave troughs might serve as beacons for FtsZ ring assembly, 43 while proteins with preferential affinity for wave crests might serve 44 to repress division. Alternatively, peptidoglycan architecture might 45 direct the formation of surface undulations and mark wave troughs 46 as future division sites. In spirochetes, peptidoglycan crosslinking 47 has been reported to impact cell shape ${ }^{21}$ and to direct the division 48 machinery to an inherited zone of active peptidoglycan synthesis ${ }^{22} .49$

Although mycobacterial wave troughs are preferred sites for cell 50 division, chromosomes also seem to play a negative regulatory role 51 in division site selection. Unlike wild-type cells, which always divide 52 within a centre-most wave trough, strains with defects in chromo- 53 some partitioning divide asymmetrically at an off-centre wave 54 trough when unpartitioned chromosomes are retained in the 55 distal cell half. These observations suggest that mycobacteria 56 might possess a mechanism analogous but not homologous to the 57 Noc system to prevent cell division over unsegregated chromo- 58 somes. Like Noc, this mechanism might serve as a 'failsafe' when 59 chromosome replication or partitioning is severely impaired ${ }^{23}$, as 60 in ParB-deficient cells. In wild-type mycobacteria, nascent septa 61 have been observed to form over chromosomes that are still in the 62 process of segregating ${ }^{24}$, and assembly of FtsZ rings over chromo- 63 somes has been observed in E. coli with diffuse nucleoids ${ }^{25}$. These 64 


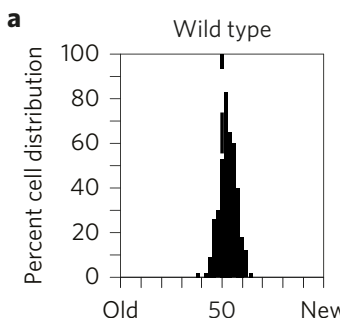

Normalized cell length

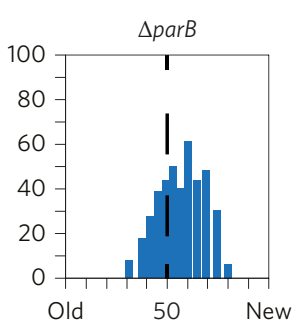

Normalized cell length b

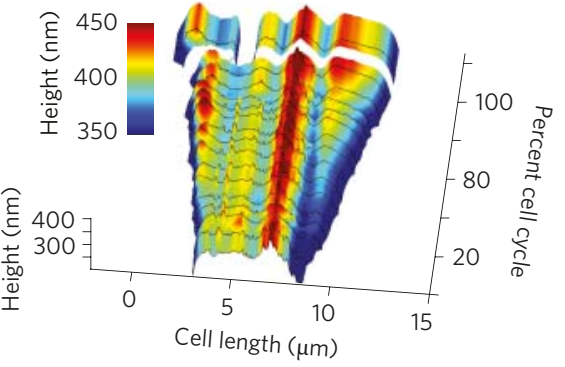

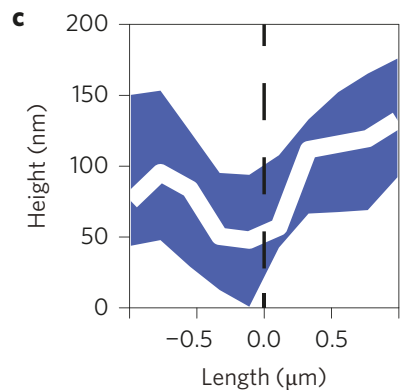

d

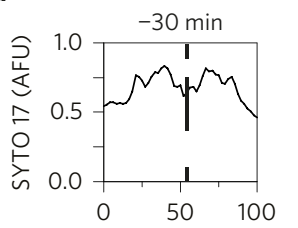

Symmetric partitioning

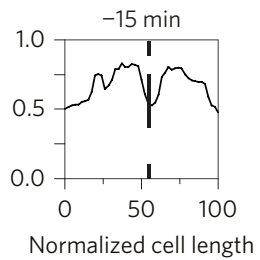

Asymmetric partitioning

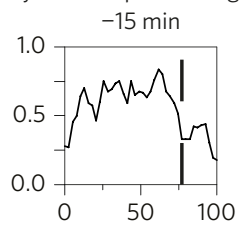

Normalized cell length
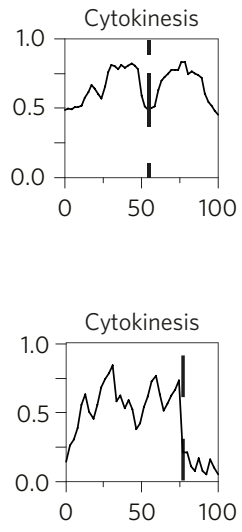

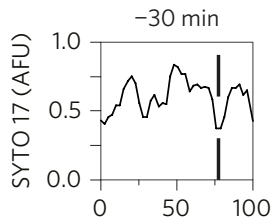

Post-ciprofloxacin

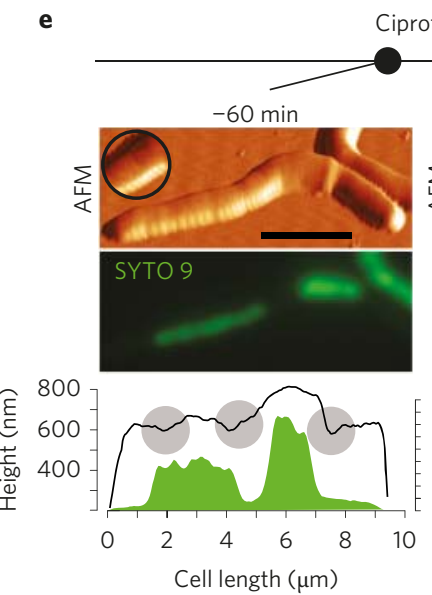

Ciprofloxacin
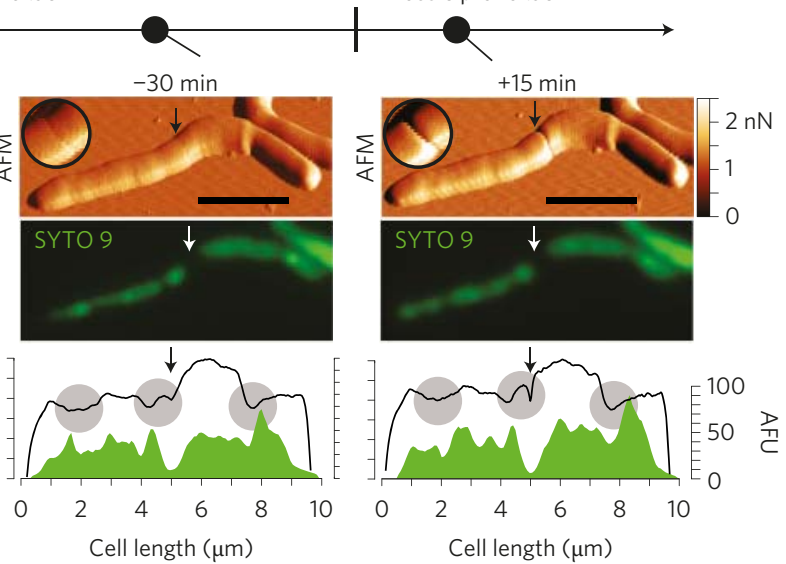

Figure 4 | Asymmetric divisions occur at off-centre wave troughs. a, Distribution of division site selection in wild-type cells (black histogram) ( $n=520$ ) and $\Delta$ parB cells (blue histogram) $(n=278)$. b. Kymograph of cell height of a representative $\Delta$ parB cell from birth to division, showing asymmetric division within an off-centre wave trough $(n=27)$. c, Averaged surface height around future sites of off-centre divisions $(n=27)$. White line: average height within a range $\pm 1 \mu \mathrm{m}$ in each direction of the cleavage site. Blue background: variation (25th and 75th percentiles) in surface height. $\mathbf{d}$, Representative graphs depicting the distribution of DNA along the cell length of $\Delta p a r B$ cells at 30, 15 and 0 min before cytokinesis. Top: examples of symmetric nucleoid partitioning ( $n=18$ ). Bottom: examples of asymmetric nucleoid partitioning leading to the formation of an anucleate new-pole daughter cell $(n=48)$. Dashed lines correspond to future division sites, which often correspond to local minima of chromosomal DNA. e, AFM (upper) and fluorescence (middle) images of a representative wild-type cell before ( -60 and $-30 \mathrm{~min}$ ) and after ( $+15 \mathrm{~min}$ ) release from a ciprofloxacin block. Longitudinal height profiles (black lines) stacked on top of DNA profiles (green plots) of the same cell show that the future division site (arrows) occurs at a local DNA minimum within a wave trough (grey shaded circle). AFM images are a three-dimensional representations of the height with the peak force error (PFE) signal overlaid as a skin. The PFE signal scale bar is expressed in nN. Scales bar (AFM images), $3 \mu \mathrm{m}$. Image sequence is representative of $n=7$.

1 observations suggest that the degree of nucleoid concentration or compaction might affect the activity of the nucleoid occlusion system. We propose a bipartite model of division site selection, in which cell-surface wave troughs are 'licensed' sites for cell division and segregated chromosomes suppress division at off-centre wave troughs. Although the molecular factors involved in division site selection in mycobacteria are unknown, the observation that cell divisions occur within wave troughs will refocus the search for such factors to a point much earlier in time than FtsZ ring formation, which is currently the earliest known event in division 10 site selection in rod-shaped bacteria.

\section{Methods} Mycobacterium smegmatis $\mathrm{mc}^{2} 155$ (wild-type) and derivative strains were 13 grown in Middlebrook 7H9 liquid medium (Difco) supplemented with $0.5 \% \quad 14$ albumin, $0.2 \%$ glucose, $0.085 \% \mathrm{NaCl}, 0.5 \%$ glycerol and $0.05 \%$ Tween- 80 . Cultures 15 were grown at $37^{\circ} \mathrm{C}$ to mid-exponential phase (optical density at $600 \mathrm{~nm}\left(\mathrm{OD}_{600}\right) \quad 16$ of $\sim 0.5$ ). Aliquots were stored in $15 \%$ glycerol at $-80{ }^{\circ} \mathrm{C}$ and thawed at room 17 temperature before use. The $\triangle$ par $B$ strain with an unmarked in-frame deletion of the 18 
parB gene has been described previously ${ }^{13}$. The attB-integrating plasmid expressing a Wag31-GFP fusion protein has also been described previously ${ }^{2}$ The Mycobacterium smegmatis RipA conditional knockdown strain was cultured and manipulated as described previously ${ }^{10}$. Wild-type cells were filamented with $500 \mathrm{ng} \mathrm{ml}^{-1}$ ciprofloxacin (Sigma) or $50 \mathrm{ng} \mathrm{ml}^{-1}$ mitomycin C (Sigma)

FtsZ-GFP reporter. The open reading frame (ORF) encoding M. smegmatis FtsZ was PCR-amplified using primers MsmftsZ-F (gctagcatgacccccccgcataactacctcg) and MsftsZ-R (ggactagttcctcctgatcctcctccacctgaaccaccaccacctgaaccaccaccacctgaaccaccggt gtgccgcatgaagggcg) with genomic DNA as the template. The underlined sequence in primer MsmftsZ-R represents the linker sequence encoding four repeats of Gly-Gly-Ser-Gly-Gly. The amplicon was ligated into vector pCR2.1 (Invitrogen) and verified by DNA sequencing, then excised as an NheI-SpeI fragment and ligated into the unique NheI site in the attB-integrating vector pND250, which encodes a hygromycin resistance marker. The resulting plasmid (pND275, provided by N. Dhar) expresses ftsZ-gfp (in-frame fusion) from a strong anhydrotetracycline (ATc)-inducible promoter. pND275 was electroporated into M. smegmatis and transformants were selected by plating on solid medium containing $50 \mu \mathrm{g} \mathrm{ml}^{-}$ hygromycin (Sigma).

19 Microscopy. For time-lapse fluorescence microscopy, bacteria were grown to mid-exponential phase $\left(\mathrm{OD}_{600} \approx 0.5\right)$ in $7 \mathrm{H} 9$ liquid medium, collected by centrifugation $(2,400 \mathrm{~g}, 5 \mathrm{~min})$, concentrated 30 -fold in fresh $7 \mathrm{H} 9$ medium $\left(37^{\circ} \mathrm{C}\right)$ and passed through a polyvinylidene difluoride syringe filter (Millipore, $5 \mu \mathrm{m}$ pore size) to remove clumps. The declumped bacteria were spread on a glass coverslip, covered with a semipermeable membrane and cultured in a custom-made microfluidic device with a continuous flow of $7 \mathrm{H} 9$ medium at $37^{\circ} \mathrm{C}$ (flow rate, $25 \mu \mathrm{min}{ }^{-1}$ ), as described previously ${ }^{26}$. Nucleoid staining was accomplished by adding SYTO 17 Red (170 nM final) in the flow medium. Bacteria were imaged at $15 \mathrm{~min}$ intervals with a DeltaVision personal DV microscope (Applied Precision) equipped with a $\times 100$ oil-immersion objective and in an environmental chamber maintained at $37^{\circ} \mathrm{C}$ (ref. 2). Images were recorded on phase-contrast and fluorescence channels (475/28 nm excitation and 525/48 nm emission filters for FITC; 575/25 nm excitation and 632/22 nm emission filters for CY-5) with a CoolSnap HQ2 camera.

AFM. Coverslips were prepared by mixing polydimethylsiloxane (PDMS) (Sylgard 184, Dow Corning) at a ratio of 15:1 (elastomer:curing agent). Air bubbles in the mixture were removed under negative pressure for $15 \mathrm{~min}$. The PDMS mixture was dropped onto a $22 \mathrm{~mm}$ glass coverslip (VWR) and spin-coated at 8,000 r.p.m. (SUSS MicroTec LabSpin6) $)^{27}$ for $30 \mathrm{~s}$. PDMS-coated coverslips were baked at $80{ }^{\circ} \mathrm{C}$ for $10 \mathrm{~min}$ before use. A $3 \mathrm{ml}$ aliquot of mid-exponential phase cell culture was filtered through a $0.5 \mathrm{~mm}$ pore size PVDF filter (Millipore) to remove cell clumps and concentrated into $200 \mathrm{ml}$ final volume by pelleting cells $(2,400 \mathrm{~g}, 8 \mathrm{~min})$. A $50 \mu \mathrm{l}$ aliquot was deposited on the hydrophobic surface of a PDMS-coated coverslip and incubated for $20 \mathrm{~min}$ to increase the surface interactions between bacteria and coverslip. A constant flow $\left(140 \mu \mathrm{l} \mathrm{min}{ }^{-1}\right)$ of $7 \mathrm{H} 9$ medium was supplied by a syringe pump. Where indicated, isoniazid (Sigma) was added to the flow medium at $5 \mu \mathrm{g} \mathrm{ml}^{-1}(1 \times \mathrm{MIC})$ or $50 \mathrm{\mu g} \mathrm{ml}^{-1}(10 \times \mathrm{MIC})$. The flow medium was preheated in a custom-made chimney that served as a bubble trap and heating element for maintaining fluid at $37^{\circ} \mathrm{C}$ in the sample space. Bacteria were imaged by a Peak Force QNM with a Nanoscope 5 controller (Veeco Metrology) at a scan rate of $0.5 \mathrm{~Hz}$ and a maximum Z-range of $5 \mu \mathrm{m}$. A ScanAsyst fluid cantilever (Bruker) was used.

Continuous scanning provided snapshots at $10 \mathrm{~min}$ intervals. Height, peak force

Q7 51 error, adhesion, dissipation, deformation, DMT modulus and log DMT modulus were recorded for all scanned images. The peak force error yields a fine representation of the height on the order of $10 \mathrm{~nm}$ in the $Z$ axis; this is computed as the difference between the peak force setpoint and the actual value. Images were processed using a custom-made MATLAB program ${ }^{28}$ or Gwyddion (Department of Nanometrology, Czech Metrology Institute). ImageJ was used for extracting bacterial cell profiles in a tabular form. MATLAB scripts were developed for automating the analysis of experimental data sets and generating graphical representations of data.

60 Correlated fluorescence and AFM. Correlated fluorescence and AFM images were acquired as described in ref. 4 . Briefly, fluorescence images were acquired with an electron-multiplying charge-coupled device (EMCCD) iXon Ultra 897 camera (Andor) mounted on an IX71 inverted optical microscope (Olympus) equipped with an UAPON100XOTIRF $\times 100$ oil immersion objective (Olympus) with the $\times 2$ magnifier in place. Illumination was provided by an MLC (monolithic laser combiner, Agilent) using the 488 or $561 \mathrm{~nm}$ laser output coupled to an optical fibre with appropriate filter sets: F36-526 for GFP and F72-866 for FM4-64 (AHF Analysetechnik). For membrane staining, $0.2 \mu \mathrm{g} \mathrm{ml}^{-1}$ FM4-64 or $15 \mu \mathrm{g} \mathrm{ml}^{-1} \mathrm{FM1}$ 43 (Life Technologies) was used. The AFM was mounted on top of the inverted microscope, and images were acquired with a Dimension Icon scan head (Bruker) using ScanAsyst fluid cantilevers (Bruker) with a nominal spring constant of $0.7 \mathrm{~N} \mathrm{~m}^{-1}$ in peak force tapping mode at a setpoint $<2 \mathrm{nN}$ and typical scan rates of $0.5 \mathrm{~Hz}$. The samples were maintained at $37^{\circ} \mathrm{C}$ in $7 \mathrm{H} 9$ liquid medium heated by a custom-made coverslip heating holder controlled by a TC2-80-150 temperature controller (Bioscience Tools).
Cell measurements

Cell growth measurements. Cell length was measured as the sum of short linear segments tracking along the midline of individual cells. Cell lengths at birth $\left(L_{\mathrm{b}}\right)$ and 78 division $\left(L_{\mathrm{d}}\right)$ were defined as distances between cell ends. Interdivision time $\left(I_{t}\right)$ was 79 defined as the time between birth and division. Elongation velocity averaged over the 80 lifetime of the cell was defined as $\left(L_{\mathrm{d}}-L_{\mathrm{b}}\right) / I_{\mathrm{t}}$. Elongation rate averaged over the 81 lifetime of the cell was defined as $\left(L_{\mathrm{d}} / L_{\mathrm{b}}\right) / I_{\mathrm{t}}$. Elongation rate averaged over a specific 82 time interval was defined as $\left(L_{\mathrm{n}} / L_{\mathrm{i}}\right) /\left(t_{\mathrm{n}}-t_{\mathrm{i}}\right)$, where $L_{\mathrm{i}}$ is the initial cell length at time $t_{\mathrm{i}} 83$ and $L_{\mathrm{n}}$ is the cell length at a later time $t_{\mathrm{n}}$. Cell volume was calculated as the sum of the 84 cylindrical volume of each incremental pixel along the midline of the cell using the 85 height as the diameter. Volumes at birth $\left(V_{\mathrm{b}}\right)$ and division $\left(V_{\mathrm{d}}\right)$ were defined for each 86 cell. The velocity of volume change averaged over the lifetime of the cell was defined 87 as $\left(V_{\mathrm{d}}-V_{\mathrm{b}}\right) / I_{\mathrm{t}}$. The rate of volume change averaged over the lifetime of the cell was 88 defined as $\left(V_{\mathrm{d}} / V_{\mathrm{b}}\right) / I_{\mathrm{t}}$. The rate of volume change averaged over a specific time interval was defined as $\left(V_{\mathrm{n}} / V_{\mathrm{i}}\right) /\left(t_{\mathrm{n}}-t_{\mathrm{i}}\right)$, where $V_{\mathrm{i}}$ is the initial cell length at time $t_{\mathrm{i}}, \quad 90$ and $V_{\mathrm{n}}$ is the cell length at a later time $t_{\mathrm{n}}$. Cell profiles were traced along the ridgeline, 91 defined as the highest point in the lateral axis following the length of the cell.

Identifying wave troughs. The longitudinal midline along the length of the cell was 93 extracted manually from the AFM height images for each individual cell at each time 94 point. To reduce the possibility of misidentifying small fluctuations between adjacent height values as waveform undulations, we applied a moving average filter 96 with an averaging window of 100-200 $\mathrm{nm}$. This smoothing treatment of the height 97 profiles did not affect the interpretation of our data, because the distances between 98 undulations are an order of magnitude greater than the smoothing window. The cell 99 profile was flattened by conducting a second-order polynomial fit of the height 100 profile (Supplementary Figs 9b and 18). The points falling below the curve fit and 101 exhibiting opposing slopes on either side are local minima. The second-order polyfit 102 localizes the wave trough position to less than $100 \mathrm{~nm}$ of the centre of a wave trough. 103 The points localized above the curve fit and exhibiting opposing slopes on either side 104 are local maxima. Wave troughs were annotated in at least two successive time points 105 within a certain spatial range corresponding to the relative increase in cell length 106 over the observed time period (see MATLAB code in file: Cell_Physiology_Analysis, 107 lines 175-179, within the Supplementary Section 'Flatten the cell height').

Identifying the central wave trough. The wave trough closest to the cell centre throughout the life of the cell is defined as the central wave trough.

Calculating the average cell surface shape at the site of cell division. Average

dimensions of the central wave trough were calculated over the interdivision time 112 (birth to cell cleavage) by averaging the flattened surface height within a range of 113 $1 \mu \mathrm{m}$ to the left and right of the division position (see MATLAB code in file: TroughProfile_Morphology). Data were collected at 197 distinct time points Troughout the interdivision times of all untreated wild-type cells. For ease of graphical representation, data from time points were binned into 15 groups. Binned 117 data were graphed in a three-dimensional surface plot.

Plotting DNA distributions and identifying anucleate daughter cells. SYTO 17-stained 119 cells were imaged by fluorescence time-lapse microscopy and dual AFM-optical 120 microscopy. Cell profiles were obtained by tracing longitudinal lines along the 121 midlines of the cells. In $\triangle p a r B$ cells, highly asymmetric divisions may lead to the 122 formation of anucleate daughter cells, which were identified as cells with very low or 123 absent DNA signals that cease to grow and divide after birth.

Data availability. Raw experimental data are available at https://figshare.com/s/ e11e1063af5cd0d02295. MATLAB scripts are accessible at https://figshare.com/s/ 3d42ad95a892c641972b.

Received 4 February 2017; accepted 9 May 2017 published XX XX 2017

\section{References}

1. Monahan, L. G., Liew, A. T., Bottomley, A. L. \& Harry, E. J. Division site 131 positioning in bacteria: one size does not fit all. Front. Microbiol. 5, 19 (2014). 132

2. Santi, I., Dhar, N., Bousbaine, D., Wakamoto, Y. \& McKinney, J. D. Single-cell 133 dynamics of the chromosome replication and cell division cycles in 134 mycobacteria. Nat. Commun. 4, 2470 (2013).

3. Fantner, G. E., Barbero, R. J., Gray, D. S. \& Belcher, A. M. Kinetics of antimicrobial peptide activity measured on individual bacterial cells using high-speed atomic force microscopy. Nat. Nanotech. 5, 280-285 (2010).

4. Odermatt, P. D. et al. High-resolution correlative microscopy: bridging the gap 139 between single molecule localization microscopy and atomic force microscopy. 140 Nano Lett. 15, 4896-4904 (2015).

5. Dufrene, Y. F. Towards nanomicrobiology using atomic force microscopy. Nat. Rev. Microbiol. 6, 674-680 (2008).

6. Dufrene, Y. F. Atomic force microscopy in microbiology: new structural and functional insights into the microbial cell surface. mBio 5, e01363-14 (2014).

\author{
(1) \\ .
}


7. Wu, Y., Sims, R. C. \& Zhou, A. AFM resolves effects of ethambutol on nanomechanics and nanostructures of single dividing mycobacteria in real-time. Phys. Chem. Chem. Phys. 16, 19156-19164 (2014).

8. Takade, A., Takeya, K., Taniguchi, H. \& Mizuguchi, Y. Electron microscopic observations of cell division in Mycobacterium vaccae V1. J. Gen. Microbiol. 129, 2315-2320 (1983).

9. Meniche, X. et al. Subpolar addition of new cell wall is directed by DivIVA in mycobacteria. Proc. Natl Acad. Sci. USA 111, E3243-E3251 (2014).

10. Hett, E. C., Chao, M. C., Deng, L. L. \& Rubin, E. J. A mycobacterial enzyme essential for cell division synergizes with resuscitation-promoting factor. PLoS Pathogens 4, el000001 (2008).

11. Mannik, J. \& Bailey, M. W. Spatial coordination between chromosomes and cell division proteins in Escherichia coli. Front. Microbiol. 6, 306 (2015).

12. Trojanowski, D. et al. Choreography of the Mycobacterium replication machinery during the cell cycle. mBio 6, e02125-e02114 (2015).

13. Santi, I. \& McKinney, J. D. Chromosome organization and replisome dynamics in Mycobacterium smegmatis. mBio 6, e01999-14 (2015).

14. Ginda, K. et al. Para of Mycobacterium smegmatis co-ordinates chromosome segregation with the cell cycle and interacts with the polar growth determinant DivIVA. Mol. Micro. 87, 998-1012 (2013).

15. Touhami, A., Jericho, M. H. \& Beveridge, T. J. Atomic force microscopy of cell growth and division in Staphylococcus aureus. J. Bacteriol. 186, 3286-3295 (2004)

16. Li, Z., Trimble, M. J., Brun, Y. V. \& Jensen, G. J. The structure of FtsZ filaments in vivo suggests a force-generating role in cell division. $E M B O$ J. 26, 4694-4708 (2007)

17. Möll, A., Schlimpert, S., Briegel, A., Jensen, G. J. \& Thanbichler, M. DipM, a new factor required for peptidoglycan remodelling during cell division in Caulobacter crescentus. Mol. Microbiol. 77, 90-107 (2010).

18. Fleurie, A. et al. MapZ marks the division sites and positions FtsZ rings in Streptococcus pneumoniae. Nature 516, 259-262 (2014).

19. Laloux, G. \& Jacobs-Wagner, C. How do bacteria localize proteins to the cell pole? J. Cell. Sci. 127, 11-19 (2014).

20. Ramamurthi, K. S., Lecuyer, S., Stone, H. A. \& Losick, R. Geometric cue for protein localization in a bacterium. Science 323, 1354-1357 (2009).

21. Huang, K. C., Mukhopadhyay, R., Wen, B., Gitai, Z. \& Wingreen, N. S. Cell shape and cell-wall organization in Gram-negative bacteria. Proc. Natl Acad. Sci. USA 105, 19282-19287 (2008).

22. Jutras, B. L. et al. Lyme disease and relapsing Borrelia elongate through zones of peptidoglycan synthesis that mark division sites of daughter cells. Proc. Natl Acad. Sci. USA 113, 9162-9170 (2016).

23. Bernhardt, T. G. \& de Boer, P. A. SlmA, a nucleoid-associated, FtsZ binding protein required for blocking septal ring assembly over chromosomes in E. coli. Mol. Cell. 18, 555-564 (2005).

24. Singh, B. et al. Asymmetric growth and division in Mycobacterium spp. compensatory mechanisms for non-medial septa. Mol. Microbiol. 88, 64-76 (2013)
25. Sun, Q. \& Margolin, W. Effects of perturbing nucleoid structure on nucleoid occlusion-mediated toporegulation of FtsZ ring assembly. J. Bacteriol. 186, 3951-3959 (2004)

26. Wakamoto, Y. et al. Dynamic persistence of antibiotic-stressed mycobacteria. Science 339, 91-95 (2013).

27. Zhang, W. Y., Ferguson, G. S. \& Tatic-Lucic, S. Elastomer-supported cold welding for room temperature wafer-level bonding. Proc. IEEE Micr. Elect. 741-744 (2004)

28. Erickson, B. W., Coquoz, S., Adams, J. D., Burns, D. J. \& Fantner, G. E. Large-scale analysis of high-speed atomic force microscopy data sets using adaptive image processing. Beilstein J. Nano. 3, 747-758 (2012).

. Lutkenhaus, J., Pichoff, S. \& Du, S. Bacterial cytokinesis: from Z ring to divisome. 59 Cytoskeleton 69, 778-790 (2012).

30. Huang, K. C. \& Ramamurthi, K. S. Macromolecules that prefer their membranes 6 curvy. Mol. Microbiol. 76, 822-832 (2010).

\section{Acknowledgements}

The authors thank S. Kondo, I. Andrews, M. Malier and O. Venzin for help in data processing and visualization. P.D.O. and G.E.F. thank A. Radenovic for discussions and the 65 use of research equipment. G.E.F. thanks C. Su and P. McPhail from Bruker Nano Surfaces Division for technical assistance. This work was funded in part by grants to J.D.M. from the Swiss National Science Foundation (310030_156945) and the Innovative Medicines Initiative (115337), a joint undertaking of the European Union Seventh Framework Programme and EFPIA (http://www.imi.europa.eu/), as well as by grants to G.E.F. from the Swiss National Science Foundation (205321 134786 and 205320 152675), the European Union FP7/2007-2013/ERC under grant agreement no. 307338-NaMic, and EU-FP7/Eurostars under award E!8213. H.A.E. was supported by a European Molecular Biology Organization Long Term Fellowship (EMBO ALTF 191-2014 and aALTF 750-2016)

\section{Author contributions}

H.A.E., J.D.M. and G.E.F. conceived the study and designed the experiments. H.A.E. P.D.O., J.X.Y.V., A.P.N. and N.D. conducted the work. H.A.E., J.D.M. and G.E.F. wrote the 78 manuscript. All authors reviewed, edited and approved the final version of the manuscript.

\section{Additional information}

Supplementary information is available for this paper.

Reprints and permissions information is available at www.nature.com/reprints.

Correspondence and requests for materials should be addressed to J.D.M. and G.E.F.

How to cite this article: Eskandarian, H. A. et al. Division site selection linked to inherited cell surface wave troughs in mycobacteria. Nat. Microbiol. 2, 17094 (2017).

Publisher's note: Springer Nature remains neutral with regard to jurisdictional claims in published maps and institutional affiliations.

\section{Competing interests}

The authors declare no competing financial interests. 
Journal: Nature Microbiology

Article ID: nmicrobiol.2017.94

Article title: Division site selection linked to inherited cell surface wave troughs in mycobacteria

Author(S): $\quad$ Haig A. Eskandarian et al.

\begin{tabular}{|c|c|}
\hline Q1 & $\begin{array}{l}\text { Author surnames have been highlighted - please } \\
\text { check these carefully and indicate if any first names } \\
\text { or surnames have been marked up incorrectly. } \\
\text { Please note that this will affect indexing of your } \\
\text { article, such as in PubMed. }\end{array}$ \\
\hline Q2 & $\begin{array}{l}\text { Reference } 11 \text { was originally cited after reference } 5 \text {, } \\
\text { so references } 6-18 \text { have been renumbered to ensure } \\
\text { a numerical order. Please check this carefully }\end{array}$ \\
\hline Q3 & $\begin{array}{l}\text { I can't see black arrows in Figure } 3 \mathrm{a}-\text { should this } \\
\text { refer to white arrowheads? }\end{array}$ \\
\hline Q4 & Please clarify what you mean by "first time point" \\
\hline Q5 & $\begin{array}{l}\text { When ambiguity may be introduced by superscript } \\
\text { references (for example after acronyms or units), } \\
\text { house style is to place them on the line, e.g. (ref. } 2 \text { ). }\end{array}$ \\
\hline Q6 & $\begin{array}{l}\text { Please expand MIC (minimum inhibitory } \\
\text { concentration?) }\end{array}$ \\
\hline Q7 & Please expand DMT \\
\hline Q8 & $\begin{array}{l}\text { I have assumed that this is in the Supplementary } \\
\text { Information. Please amend if this is incorrect. }\end{array}$ \\
\hline Q9 & $\begin{array}{l}\text { Please note that refs. } 29,30 \text { are listed in refs list but } \\
\text { not cited in the text. Please indicate where they } \\
\text { should be cited, or delete the refs from the ref list. }\end{array}$ \\
\hline Q10 & Please check grant number E! 8213 \\
\hline Q11 & Please check grant number aALTF 750-2016 \\
\hline Q12 & $\begin{array}{l}\text { In the Author contributions, author M.T.M.H. is not } \\
\text { mentioned - is this intentional? Please check and, if } \\
\text { not, please provide a contribution for this author. }\end{array}$ \\
\hline Q13 & $\begin{array}{l}\text { Fig } 4 d \text { - please provide expanded form of units } \\
\text { AFU }\end{array}$ \\
\hline
\end{tabular}

